\title{
Study on the Development of Decision Making Using Intuitionistic Fuzzy Set (IFS) and Interval Valued Intuitionistic Fuzzy Set (IVIFS)
}

\author{
Yasir Ahmad ${ }^{1}$, Sadia Husain ${ }^{2}$, Irfan Sayeed Asthanvi ${ }^{3}$ \\ 1,2,3 (Faculty of Computer Science, Jazan University, K.S.A.)
}

\begin{abstract}
Out of several higher order fuzzy sets [1], the concept of an intuitionistic fuzzy set (IFS) [2] introduced by Atanassov has been found to be highly useful to deal with vagueness and imprecision. IFS theory has been extensively applied to areas like Artificial Intelligence, networking, Soft decision making, Programming logic, operational research etc. One the promising role of IFS has been emerged in Decision making problems specially group decision making and multi-attribute decision making. In some real-life situations, decision makers may not be able to accurately express their view for the problem as they may not possess a precise or sufficient level of knowledge of the problem or the decision makers are unable to discriminate explicitly the degree to which one alternative are better than others in such cases, the decision maker may provide their preferences for alternatives to a certain degree, but it is possible that they are not so sure about it [3]. Thus, it is very suitable to express the decision maker preference values with the use of fuzzy/intuitionistic fuzzy values rather than exact numerical values or linguistic variables [4]. To satisfy the need of decision making problem with imprecision and uncertainty many researchers have been concentrated on IFS theory. In this paper we reviewed the development of different approaches for solving decision making problem using IFS theory and a brief introduction on the role of Interval Valued IF sets (IVIFS) [5] in multiattribute decision making.
\end{abstract}

Keywords: Intuitionistic fuzzy sets (IFS), Multicriteria Decision Making (MCDM), Interval Valued Intuitionistic fuzzy sets (IVIFS)

\section{INTRODUCTION}

The classical decision making methods generally assume that all criteria and their respective weights are expressed in crisp values and, thus, that the rating and the ranking of the alternatives can be carried out without any problem. In a real-world decision situation, the application of the classical decision making method may face serious practical constraints from the criteria perhaps containing imprecision in the information. In many cases, performance of the criteria can only be expressed qualitatively or by using linguistic terms, which certainly demands a more appropriate method. The most preferable situation for a decision making problem is when all ratings of the criteria and their degree of importance are known precisely, which makes it possible to arrange them in a crisp ranking. However, many of the decision making problems in the real world take place in an environment in which the goals, the constraints, and the consequences of possible actions are not known precisely (Bellman and Zadeh, 1970). As a result, the best condition for a classic decision making problem may not be satisfied, when the decision situation involves both fuzzy and crisp data. The classical decision making methods cannot handle such problems effectively, because they are only suitable for dealing with problems in which all performances of the criteria are represented by crisp numbers. The application of the fuzzy set theory in the field of decision making is justified when the intended goals or their attainment cannot be defined or judged crisply but only as fuzzy sets (Zimmermann, 1987).

The concept of an intuitionistic fuzzy set can be viewed as an alternative approach to define a fuzzy set in cases where available information is not sufficient for the definition of an imprecise concept by means of a conventional fuzzy set. In general, the theory of intuitionistic fuzzy sets is the generalization of fuzzy sets. Therefore, it is expected that intuitionistic fuzzy sets could be used to simulate human decision-making processes and any activities requiring human expertise and knowledge which are inevitably imprecise or not totally reliable. In year 1989 Atanassov introduced Interval-valued intuitionistic fuzzy sets and many researchers have shown interest in the IVIFS theory and successfully applied it to the field of multi-criteria decision making. In this paper, we will also give a brief review on the contribution of IVIFS in the field of multi-criteria decision making. In this paper, we will review almost all the essential contributions done in decision making methods based on intuitionistic fuzzy set theory. Decision making can be a group decision making and it can be characterized by multicriteria (MCDM) or multiperson [6]. MCDM is concerned with structuring and solving decision and planning problems involving multiple criteria. Typically, there does not exist a unique optimal solution for such problems and it is necessary to use decision maker's preferences to 
differentiate between solutions. Multiple criteria decision making (MCDM) is often use for dealing with complex engineering problems and IFS theory has been extensively applied to it.

This paper is organized as follows. The definitions of intuitionistic fuzzy sets and Interval-valued IF are introduced in Section 2. In Section 3 we discuss the role of IFS in simple decision making. Multi-criteria decision-making models with intuitionistic and interval-valued fuzzy sets are reviewed, and their developments are established in Section 4 and 5, finally conclusion is given in Section 6.

\section{DEFINITIONS OF INTUITIONISTIC FUZZY SETS}

Let a set $\mathrm{E}$ be fixed. An IFS $\mathrm{A}$ in $\mathrm{E}$ is an object of the following form [8]. $\mathrm{A}=\left\{\left(\mathrm{x}, \mu_{\mathrm{A}}(\mathrm{x}), v_{\mathrm{A}}(\mathrm{x})\right)\right.$ $\mathrm{x} \in \mathrm{E}\}$, Where the functions $\mu_{\mathrm{A}}(\mathrm{x}): E \rightarrow[0,1]$ and $v_{\mathrm{A}}(\mathrm{x}): E \rightarrow[0,1]$ determine the degree of membership and the degree of non-membership of the element $\mathrm{x} \in \mathrm{E}$, respectively, and for every $\mathrm{x} \in \mathrm{E}$ :

$0 \leq \mu_{\mathrm{A}}(\mathrm{x})+v_{\mathrm{A}}(\mathrm{x}) \leq 1$

When $v_{A}(\mathrm{x})=1-\mu_{\mathrm{A}}(\mathrm{x})$ for all $x \in \mathrm{E}$ is ordinary fuzzy set. In addition, for each IFS $\mathrm{A}$ in $\mathrm{E}$, if

$\pi_{\mathrm{A}}(\mathrm{x})=1-\mu_{\mathrm{A}}(\mathrm{x})-v_{\mathrm{A}}(\mathrm{x})$

Then $\pi_{\mathrm{A}}(\mathrm{x})$ is called the degree of indeterminacy of $\mathrm{x}$ to $\mathrm{A}$ or called the degree of hesitancy of $\mathrm{x}$ to A. It is obvious that

$0 \leq \pi_{\mathrm{A}}(\mathrm{x}) \leq 1 ;$ For each $x \in \mathrm{E}$

Especially, if $\pi_{\mathrm{A}}(\mathrm{x})=0$, for all $\mathrm{x} \in \mathrm{E}$ then the IFS $\mathrm{A}$ is reduced to a fuzzy set.

On the other hand, for each fuzzy set $\mathrm{A}^{\prime}$ in $\mathrm{E}$, we evidently have: $\quad \pi_{\mathrm{A}^{\prime}}(\mathrm{x})=1-\mu_{\mathrm{A}^{\prime}}(\mathrm{x})-\left[1-\mu_{\mathrm{A}^{\prime}}(\mathrm{x})\right]$

Therefore, if we want to fully describe an intuitionistic fuzzy set, we must use any two functions from the triplet:

- membership function,

- non-membership function,

- hesitation margin

In other words, the application of intuitionistic fuzzy sets instead of fuzzy sets means the introduction of another degree of freedom into a set description (i.e. in addition $\mu_{\mathrm{A}(\mathrm{x})}$ to we also $v_{\mathrm{A}}$ or $\pi_{\mathrm{A}}$ ). Since the intuitionistic fuzzy sets being a generalization of fuzzy sets give us an additional possibility to represent imperfect knowledge, they can make it possible to describe many real problems in a more adequate way.

\section{DEFINITIONS INTERVAL-VALUED INTUITIONISTIC FUZZY SETS}

Sometime it is not appropriate to assume that the membership degrees for certain elements of A are exactly defined, but a value range can be given. In such cases, Atanassov and Gargov defined the notion of interval-valued intuitionistic fuzzy set (IVIFS) as below:

Let $X=\{x 1, x 2, x 3 \ldots x n\}$ be a given set and $D[0,1]$ be the set of all closed subintervals of the interval $[0,1]$, and $\mathrm{X}$ be ordinary finite non-empty sets. An interval valued intuitionistic fuzzy set $\mathrm{A}$ in $\mathrm{X}$ is an expression given by

$A=\left\{<\tilde{x}, \tilde{\mu}_{\tilde{A}}(x)+\tilde{v}_{\tilde{A}}(x)>x \in X\right\}$

Where

$\tilde{\mu}_{\tilde{A}}: X \rightarrow D[0,1], \tilde{v}_{\tilde{A}}: X \rightarrow D[0,1]$

with the condition $\sup \left(\tilde{\mu}_{\tilde{A}}(\mathrm{x})\right)+\sup \left(\tilde{v}_{\tilde{A}}(\mathrm{x})\right) \leq 1$

Especially, if each of the intervals $\mu_{\tilde{A}}(\mathrm{x})$ and $v_{\tilde{A}}(\mathrm{x})$ contains exactly one element, i.e., if for every ${ }_{\mathrm{x}} \in \mathrm{X}$

$\mu_{\tilde{A}}(x)=\operatorname{INF}\left(\mu_{\tilde{A}}(x)\right)=\sup \left(\mu_{\tilde{A}}(x)\right)$,

$v_{\tilde{A}}(x)=\operatorname{INF}\left(v_{\tilde{A}}(x)\right)=\sup \left(v_{\tilde{A}}(x)\right)$

then, the given IVIFS $\tilde{A}$ is transformed to an ordinary intuitionistic fuzzy set Based on IVIFS, Xu [7] defined the notion of interval-valued intuitionistic fuzzy number (IVIFN):

Definition: Let $\tilde{A}=\left\{<\tilde{x}, \tilde{\mu}_{\tilde{A}}(x)+\tilde{v}_{\tilde{A}}(x)>x \in X\right\}$, be an IVIFS, then we call the pair $\left(\tilde{\mu}_{\tilde{A}}(x)+\tilde{v}_{\tilde{A}}(x)\right)$ an IVIFN.

\section{ROLE OF IFS IN DECISION MAKING PROBLEMS}

Fuzzy set theory has been used for handling fuzzy decision-making problems [8-12] for a long span of time but many researchers have shown interest in the IFS theory and applied it to the field of decision making. Gau and Buehrer [13] introduced the vague set [14], which is an equivalence of IFS. Later, based on vague sets, Chen and Tan [15], and Hong and Choi [16] utilized the minimum and maximum operations to develop some approximate technique for handling multi-attribute decision making problems under fuzzy environment.

In year 1995 [17] Attanasov et al. worked on a project titled "Decision making methods based on element of Intuitionistic Fuzzy Sets and their Software Implementation", the basic aim of the project was to envisage following four activities. 
A1: Theoretical research related to the construction and description of some new decision making methods.

A2: Methodological research for the use of these methods in practice.

A3: Development of software implementation of decision making methods from A1

A4: Construction of examples for the application of decision making methods from A1 in medicine.

Later on in year 1999 [18] Atanassov also discussed the possibility of using the interpretational triangle of intuitionistic fuzzy set in decision making. But the major contribution in this field comes from Szmidt and Kacprzyk [19-20] who intensively worked on the use of intuitionistic fuzzy sets for building soft decisionmaking models. They proposed two solution concepts about the intuitionistic fuzzy core and the consensus winner for group decision making. The concept of preference relation was considered by many authors, in the crisp case for example in [21] and in the fuzzy environment [22]. Szmidt and Kacprzyk [23] were also the first authors who generalized the concept of preference relation from the fuzzy case to the intuitionistic fuzzy one. They take into account intuitionistic fuzzy preference relations which are applied in group decision making problems where a solution from the individual preferences over some set of options should be derived. In year 2002 they used a new approach and calculate distance between intuitionistic fuzzy preferences to assess agreement of a group of experts [24]. In another article [25] they propose how to analyze the extent of agreement in a group of experts employing intuitionistic fuzzy sets. They used the concept of distances between intuitionistic fuzzy preferences as the main tool to evaluate how far the group is from full agreement (consensus in a traditional sense) and they also propose how to evaluate if it is possible for a considered group to come closer to the state of consensus. They used Entropy as the measure which makes it possible to say how strong the preferences of experts are.

Recently in year 2012 Szmidt et al. [26] further worked on IF relation and they present a construction method for Atanassov's intuitionistic fuzzy preference relations starting from fuzzy preference relations and taking into account the ignorance of the expert in the construction of the latter. Moreover, they also propose two generalizations of the weighted voting strategy to work with Atanassov's intuitionistic fuzzy preference relations. An advantage of these algorithms is that they start from fuzzy preference relations and their results can be compared with those of any other decision making. They also worked on similarity measure [27] of IF sets and proposed a measure that takes into account not only a pure distance between intuitionistic fuzzy preferences but also examines if the compared preferences are more similar or more dissimilar. The agreement of a whole group is assessed via an aggregation of individual testimonies expressed by Intuitionistic fuzzy preference relations.

Zeshui $\mathrm{Xu}$ [28] also worked on Intuitionistic preference relations and their application in group decision making. He developed an approach to group decision making based on intuitionistic preference relations and an approach to group decision making based on incomplete intuitionistic preference relations respectively, in which the intuitionistic fuzzy arithmetic averaging operator and intuitionistic fuzzy weighted arithmetic averaging operator are used to aggregate intuitionistic preference information, and the score function and accuracy function are applied to the ranking and selection of alternatives. Later on in year 2009 Zeshui Xu et al. [29] studied the IF similarity measure introduced by Szmidt and Kacprzyk and find it somewhat inconvenient in some cases, and thus they develop a new similarity measure between intuitionistic fuzzy sets.

Z. Gong [30] in year 2007 investigates the problem of group decision making based on intuitionistic fuzzy judgment matrix. They introduced the ranking method of intuitionistic fuzzy judgment matrix by using operations of intuitionistic fuzzy values and illustrated their method by a numerical example.

Cuiping Wei and Xijin Tang [31] in year 2011 study group decision-making problems based on intuitionistic preference relations. By measuring the uncertain information of intuitionistic preference relations and the average similarity degree of one individual intuitionistic preference relation to the others, they proposed a new approach to assess the relative importance weights of experts. Their approach takes both the objective and subjective information of experts into consideration. Then they integrate the weights of experts into the individual intuitionistic preference relations and develop a relative similarity method to derive the priorities of alternatives.

Zeshui Xu and M. Xia [32-33] used entropy and cross entropy and gave two methods to determine the optimal weights of attributes, and develop two pairs of entropy and cross entropy measures for intuitionistic fuzzy values. They also discussed the properties of these measures and the relations between them and the existing ones. Furthermore, they introduce three new aggregation operators, which treat the membership and non-membership information fairly, to aggregate intuitionistic fuzzy information.

Yejun $\mathrm{Xu}$ and Huimin Wang [34] presented the induced generalized intuitionistic fuzzy ordered weighted averaging (I-GIFOWA) operator. It is a new aggregation operator that generalized the IFOWA operator, including all the characteristics of both the generalized IFOWA and the induced IFOWA operators. It provides a very general formulation that includes as special cases a wide range of aggregation operators for intuitionistic fuzzy information, including all the particular cases of the I-IFOWA operator, GIFOWA operator and the induced intuitionistic fuzzy ordered geometric (I-IFOWG) operator. 


\section{IFS AND MULTICRITERIA DECISION MAKING (MCDM)}

Cenigiz Kahraman gave very useful description about MCDM in his book on Fuzzy Multicriteria Decision Making [35]. In his book he explained MCDM problems with two basic approaches: multiple attribute decision making (MADM) and multiple objective decision making (MODM). MADM problems are distinguished from MODM problems, which involve the design of a "best" alternative by considering the tradeoffs within a set of interacting design constraints. MADM refers to making selections among some courses of action in the presence of multiple, usually conflicting, attributes. In MODM problems, the number of alternatives is effectively infinite, and the tradeoffs among design criteria are typically described by continuous functions. MADM approaches can be viewed as alternative methods for combining the information in a problem's decision matrix together with additional information from the decision maker to determine a final ranking, screening, or selection from among the alternatives. Besides the information contained in the decision matrix, all but the simplest MADM techniques require additional information from the decision maker to arrive at a final ranking, screening, or selection. It has been widely recognized that most decisions made in the real world take place in an environment in which the goals and constraints, because of their complexity, are not known precisely, and thus, the problem cannot be exactly defined or precisely represented in a crisp value (Bellman and Zadeh, 1970). Bellman and Zadeh (1970) and Zimmermann (1978) introduced fuzzy sets into the MCDM field. They cleared the way for a new family of methods to deal with problems that had been inaccessible to and unsolvable with standard MCDM techniques. Bellman and Zadeh (1970) introduced the first approach regarding decision making in a fuzzy environment.

In year 1995 Humberto Bustince [36] presented a new technique for handling decision making problems based on intuitionistic fuzzy sets. In this new technique he used the degree of reliability and of nonreliability of each criterion in relation to a set of alternatives. He also presented a new score function in order to evaluate the degree of suitability of the choice of a certain alternative. Since then lots of research has been made in the field of multicriteria decision making using IFS.

In year 2002 [37] Atanassov et al. proposed an IF interpretation of multi-person multi-criteria decision making. In this work each expert is asked to evaluate each alternative in terms of its performance with respect to each predefined criterion. The experts evaluations are expressed as a pair of numeric values, interpreted in the intuitionistic fuzzy framework, theses number express a positive and negative evaluation respectively. Each expert is also assigned a pair of values which express expert's reliability. They further extended this work in year 2003 and 2004 [38]. They constructed a Generalized Net model of Multi-Person Multi-Criteria Decision Making process based on IF graph. In year 2005 they also introduced an IF interpretation of the processes of multi-person and multi measurement tool multi-criteria decision makings 2005[39].

In year 2006 many authors worked on multicriteria decision making problems some of them are:

- Eulalia Szmidt and Janusz Kacprzyk [40] proposed a new solution to a multi-criteria decision making problem by using similarity measures for intuitionistic fuzzy sets. They show that the new solution is better than the method proposed by Chen and Tan [41] on vague set which fails in some situations.

- Lin et al. [42] proposed a method that allows the degrees of satisfiability and non-satisfiability of each alternative with respect to a set of criteria to be represented by intuitionistic fuzzy sets, respectively. Furthermore, their proposed method allows the decision-maker to assign the degrees of membership and nonmembership of the criteria to the fuzzy concept "importance." This method can provide a useful way to efficiently help the decision-maker to make his decision.

- Liu and Wang [43] introduce and discuss the concept of intuitionistic fuzzy point operators. The intuitionistic fuzzy point operators can reduce the degree of uncertainty of the elements in a universe corresponding to an intuitionistic fuzzy set. Furthermore, they defined a series of new score functions for multi-criteria decisionmaking problem based on the intuitionistic fuzzy point operators and the evaluation function and their effectiveness and advantage are illustrated by examples.

\section{INTERVAL VALUED IFS IN DECISION MAKING PROBLEMS}

Fuzzy set theory has been used for handling fuzzy decision-making problems for a long span of time but many researchers have shown interest in the IFS theory and applied it to the field of decision making. But after the introduction of IVIFS, there is a shift from IFS to IVIFS and researcher finds that Interval-valued intuitionistic fuzzy set (IVIFS) is effective in dealing with fuzziness and uncertainty inherent in decision data and multi-attribute decision making (MADM). In year 2006 Chunqiao Tan and Qiang Zhang [44] presented a novel method for multiple attribute decision-making based on interval valued intuitionistic fuzzy sets (IVIFSs) theory and TOPSIS method in fuzzy environments. In their paper, the concept of interval-valued intuitionistic fuzzy sets is introduced, and the distance between two interval valued intuitionistic fuzzy sets is defined. Then, according to the ideal of classical TOPSIS method, a closeness coefficient is defined to determine the ranking order of all alternatives by calculating the distances to both the interval valued intuitionistic fuzzy positive-ideal 
solution and interval valued intuitionistic fuzzy negative-ideal solution. The multi-attribute decision-making process based on IVIFSs is given in fuzzy environments.

In year 2007, Dynamic intuitionistic fuzzy multi-attribute decision making was introduced by Zeshui et al. [45]. They investigate dynamic multi-attribute decision making problems with intuitionistic fuzzy information. The notions of intuitionistic fuzzy variable and uncertain intuitionistic fuzzy variable are defined, and two new aggregation operators: dynamic intuitionistic fuzzy weighted averaging (DIFWA) operator and uncertain dynamic intuitionistic fuzzy weighted averaging (UDIFWA) operator are presented. Some methods, including the basic unit-interval monotonic (BUM) function based method, normal distribution based method, exponential distribution based method and average age method, are introduced to determine the weight vectors associated with these operators. A procedure based on the DIFWA operator is developed to solve the dynamic intuitionistic fuzzy multi-attribute decision making (DIF-MADM) problems where all the decision information about attribute values takes the form of intuitionistic fuzzy numbers collected at different periods, and a procedure based on the UDIFWA operator is developed for DIF-MADM under interval uncertainty in which all the decision information about attribute values takes the form of interval-valued intuitionistic fuzzy numbers collected at different periods. Another article on DIF-MADM is given by Si Yan-jie and Wei Fa-jie [46].

In year 2007 Jun Ye [47] investigated aggregating interval-valued intuitionistic fuzzy sets (IVIFSs). Some operational laws of IVIFSs are defined. He proposed some aggregation operators, including intervalvalued intuitionistic fuzzy weighted arithmetic average operator and interval-valued intuitionistic fuzzy weighted geometric average operator. To identify the best alternative in multicriteria decision-making problems, a multicriteria fuzzy decision-making method based on the correlation coefficient of IVIFSs is established in which criterion values for alternatives are IVIFSs. He also utilize the interval-valued intuitionistic fuzzy weighted aggregation operators to aggregate the interval-valued intuitionistic fuzzy information corresponding to each alternative, and then rank the alternatives and select the most desirable one(s) according to correlation coefficients between the alternatives and the ideal alternative.

In year 2008 many authors contributed in this area but the work of some of them are more significant and they are:

Guiwu Wei and Wende Yi [48] proposed a new decision making analysis method based on the minimum deviation. They utilize the intuitionistic fuzzy weighted averaging (IFWA) operator to aggregate the intuitionistic fuzzy information corresponding to each alternative, and then rank the alternatives and select the most desirable one(s) according to the score function and accuracy function. The method can sufficiently utilize the objective information, and meet decision maker's subjective preference.

Ludmila Dymova et al. [49-50] in their research analyzed that the main problem of known methods for Multiple Criteria Decision Making in the Intuitionistic Fuzzy setting is that they are generally based on the intermediate type reduction. Such approaches lead inevitable to the loss of important information. Another problem is the choice of an appropriate method for the local criteria aggregation taking into account their ranks. The aim of their paper is to present a new method which makes it possible to solve the first problem and facilitates the solution of the second one. The method is based on the Dempster-Shafer Theory (DST). It allows solving the Multiple Criteria Decision Making problem without intermediate type reduction for different approaches to aggregation of the local criteria.

Zhoujing Wang et al. [51] in 2008 used interval-valued intuitionistic fuzzy matrices, interval-valued intuitionistic fuzzy number and developed several optimization models to generate optimal weight of the attribute and the corresponding decision making methods.

In year 2009 Szmidt and Kacprzyk [52] propose a new solution to a ranking oriented multi-criteria decision making problem for the alternatives expressed via Atanassov's intuitionistic fuzzy sets. They show that the new solution better fulfills our expectations than methods proposed by Chen and Tan's[15].

In year 2009, Z. Wang et al. [53] By employing a projection model, fractional programming models are developed to determine the closeness interval values of alternatives. The interval values are subsequently used to aggregate into an overall interval value for each alternative, and the likelihood is applied to ranking and selection of alternatives. Another article on the use of projection method is given by Yujun Luo [54].

Yujun Luo [55] in year 2009 introduced the decision making matrix with the attribute value expressed by form of intuitionistic fuzzy number further transformed it into the score matrix of the alternatives. Then according to the concept of regret, regret matrix of the alternatives is obtained. And an optimization model based on the principle of minimization of regret, by which the attribute weights can be derived, is established. The alternatives can be ranked, and the most desirable one can be selected according to the score function and accuracy function.

Juan Li and Chengyi Zhang [56] gave a method to choose the possible positive-ideal solution and the possible negative-ideal solution, and deal with the uncertainty information by entropy theory to get the only ideal alternatives. They also introduced a new math model for solving the best attribute weight vector based on 
the isomorphism of intuitionistic fuzzy sets, and the process for solving multiple attribute decision-making is also introduced.

Fatih Emre et al. proposed TOPSIS method combined with intuitionistic fuzzy set to select appropriate supplier in group decision making environment. Intuitionistic fuzzy weighted averaging (IFWA) operator is utilized to aggregate individual opinions of decision makers for rating the importance of criteria and alternatives. Other articles based on Supplier Selection Method are given by Hongjun [57] and Lei Wen, et al. [58].

Luo Yongbiao et al. [59] used the weighted correlation coefficient of interval-valued intuitionistic fuzzy sets (IVIFSs) to identify the best alternative in multicriteria decision-making.

Weibo Lee [60] also gave an enhanced multicriteria decision-making method of machine design schemes under interval-valued intuitionistic fuzzy environment.

In year 2010 Gui-Wu Wei [61] established an optimization model based on the basic ideal of traditional grey relational analysis (GRA) method, by which the attribute weights can be determined. Then, based on the traditional GRA method, calculation steps for solving intuitionistic fuzzy multiple attribute decision-making problems with incompletely known weight information are given. The degree of grey relation between every alternative and positive-ideal solution and negative-ideal solution are calculated. Then, a relative relational degree is defined to determine the ranking order of all alternatives by calculating the degree of grey relation to both the positive-ideal solution (PIS) and negative-ideal solution (NIS) simultaneously. Another article based on GRA is given by Xiangqian Feng and Gang Qian [62].

Deng-Feng Li [63-64] developed a Linear and nonlinear-programming methodology that is based on the technique for order preference by similarity to ideal solution to solve multiattribute decision-making (MADM) problems with both ratings of alternatives on attributes and weights of attributes expressed with IVIF sets.

In year 2011 Jian-Zhang Wu and Qiang Zhang [65] introduce some classical intuitionistic fuzzy entropy measures and verify the entropy of the intuitionistic fuzzy set is the average value of the entropies of its intuitionistic fuzzy values. Then, they present the concept of the intuitionistic fuzzy weighted entropy, which is a natural extension of the entropy for intuitionistic fuzzy sets. According to the minimum entropy principle, they establish the programming model to generate optimal weight of criteria, and present the corresponding multicriteria decision making method. Xiaohan $\mathrm{Yu}$ et al. [66] investigate hybrid multiple attribute decision making problems with various forms of attribute values (real numbers, linguistic labels, interval numbers, intuitionistic fuzzy numbers and interval intuitionistic fuzzy numbers). They proposed a method based on preference degrees which may take the forms of fuzzy numbers, intuitionistic fuzzy numbers and interval intuitionistic fuzzy numbers. The method first normalizes various forms of attribute values into preference degrees, and then uses a preference degree-based weighted averaging operator to aggregate the normalized preference degrees. A new linguistic representation model is presented, whose feasibility is verified by comparing it with the traditional 4-tuple linguistic representation model, and from their model, the mapping relationship between interval intuitionistic fuzzy numbers and linguistic labels can be constructed. Zhao Zhitao and Zhang Yingjun [67] developed a method that is based on the accuracy function to solve MADM problems with both ratings of alternatives on attributes and weights of attributes expressed with IVIFSs. Some other researcher like ZuBei Ying et al. [68] and Ting-Yu Chen et al. [69] also worked on multiple attribute group decision making based on interval-valued intuitionistic fuzzy sets.

Guiwu Wei and Xiaofei Zhao [70] in February 2012 introduced some operational law, score function and accuracy function of intuitionistic fuzzy values. Then two new aggregation operators: induced intuitionistic fuzzy correlated averaging (I-IFCA) operator and induced intuitionistic fuzzy correlated geometric (I-IFCG) operator are developed and some desirable properties of the I-IFCA and I-IFCG operators are studied, such as commutative, idempotency and monotonicity. An I-IFCA and IFCA (intuitionistic fuzzy correlated averaging) operators-based approach is developed to solve the MAGDM problems in which both the attribute weights and the expert weights usually correlative, attribute values take the form of intuitionistic fuzzy values.

Zeshui $\mathrm{Xu}$ and Meimei Xia [71] in their paper, first transformed the original decision matrix, whose elements are intuitionistic fuzzy numbers expressed by pairs of satisfaction degrees and dissatisfaction degrees, into its expected decision matrix, whose elements are composed of satisfaction degrees and hesitation degrees. Then they introduced the concept of dominated alternative, and give a method to identify the dominated alternatives. Then they developed an interactive method for eliminating any dominated alternatives by updating the decision maker's preferences gradually so as to find out the optimal one eventually.

In year 1212 only few researchers worked in this area, Jian-qiang et al. [72] in their article analyze the limitations of existing score functions of intuitionistic fuzzy set. They introduced a new score function based on the prospect value function and used this, the prospect score function to develop an interval-valued intuitionistic fuzzy multi-criteria decision-making approach. This approach gives a matrix of score function values and a comprehensive evaluation value of each alternative. 


\section{CONCLUSION}

In this paper we have presented a review on the role of Intuitionistic and interval-valued fuzzy set theory in decision making problems. As we know that decision makers face many problems with incomplete and vague information in decision making problems since the characteristics of these problems often require this kind of information. So Intuitionistic fuzzy/ Interval valued approaches are suitable to use when the modeling of human knowledge is necessary and when human evaluations are needed. Considering this as an important issue many researchers have paid attention in this area. In this paper we have reviewed all the important contribution in this area by different authors. We have also given the brief introduction on the contribution of Interval valued Intuitionistic fuzzy set with MADM and all the important approaches, methods used and introduced by the researchers in their paper.

\section{REFERENCES}

[1]. L. A. Zadeh, "Fuzzy sets", Information and Control 8 (3) (1965) 338-353.

[2]. K.T. Atanassov, "Intuitionistic fuzzy sets", Fuzzy Sets and Systems 20 (1986) 87-96.

[3]. F. Herrera, L. Martinez, P.J. Sanchez, "Managing non-homogeneous information in group decision making", European Journal of Operational Research 166 (2005) 115-132.

[4]. E. Szmidt, J. Kacprzyk, "Using intuitionistic fuzzy sets in group decision making", Control and Cybernetics 31 (2002) 1037-1053.

[5]. K.T. Atanassov, G.Gargov, Interval valued intuitionistic fuzzy sets [J],Fuzzy Sets and Systems. 1989, 31, 343-349.

[6]. L.I. Dengfeng, "Fuzzy Multi objective Many-Person Decision Makings and Games", National Defense Industry Press, Beijing, 2003.

[7]. Ze. Xu, J. Chen, Approach to Group Decision Making Based on Interval-Valued Intuitionistic Judgment Matrices, Systems Engineering - Theory \& Practice, Volume 27, Issue 4, April 2007, Pagesiu 126-133.

[8]. S.M. Chen, "A new approach to handling fuzzy decision making problems", IEEE Transactions on Systems Man and Cybernetics 18 (1988) 1012-1016.

[9]. R. Bellman, L.A. Zadeh, "Decision making in a fuzzy environment", Management Science 17 (1970) $141-164$.

[10]. W.J.M. Kickert, "Fuzzy Theories on Decision Making: A Critical Review", Kluwer, Boston, 1978.

[11]. H.J. Zimmermann, "Fuzzy Sets, Decision Making, and Expert Systems", Kluwer Academic Publishers, Boston, 1987.

[12]. Lang, C. Hwang, K. Yoon, "Multiple Attribute Decision Making: Methods and Applications", SpringerVerlag, New York, 1981.

[13]. W.L. Gau, D.J. Buehrer, "Vague sets", IEEE Transactions on Systems, Man, and Cybernetics 23 (1993) 610-614.

[14]. H. Bustine, P. Burillo, "Vague sets are intuitionistic fuzzy sets", Fuzzy Sets and Systems 79 (1996) $403-$ 405.

[15]. S.M. Chen, J.M. Tan, "Handling multicriteria fuzzy decision-making problems based on vague set theory", Fuzzy Sets and Systems 67 (1994) 163-172.

[16]. D.H. Hong, C.H. Choi, "Multicriteria fuzzy decision-making problems based on vague set theory", Fuzzy Sets and Systems 114 (2000)103-113.

[17]. P. Angelov, O. Asparoukhov, K. Atanassov, P.B. Lopez, H.B. Sola, S. Hadjitodorov, S.K. Kim, J. Kacprzyk, L. Kuncheva, A. Shannon "Decision making methods based on elements of intuitionistic fuzzy sets and their software implementation", Notes on IFS, Volume 1 (1995) Number 1, pages 40-41.

[18]. C. Temponi, A. Shannon, Atanassov K., A. Ban, "An idea for an intuitionistic fuzzy approach to decision making"," Notes on IFS", Volume 5 (1999) Number 3, pages 6-10.

[19]. E. Szmidt, J. Kacprzyk, "Intuitionistic fuzzy sets in group decision making", NIFS 2 (1) (1996) 15-32.

[20]. E. Szmidt, J. Kacprzyk, "Remarks on some applications of intuitionistic fuzzy sets in decision making", NIFS 2 (3) (1996)22-31.

[21]. M. Roubens and P. Vincke, editors. "Preference Modeling", Springer-Verlag, Berlin, 1985

[22]. F. Chiclana, E. Herrera-Viedma, S. Alonso, R. A. M. Pereira, "Preferences and consistency issues in group decision making. In H. Bustince at al., editors", Fuzzy Sets and Their extensions: Representation, Aggregation and Models, Springer-Verlag, Berlin, (2008) 219-237.

[23]. E. Szmidt and J. Kacprzyk, "Using intuitionistic fuzzy sets in group decision making", Control and Cybernetics 31, (2002)1037-1053.

[24]. E. Szmidt and J. Kacprzyk "Evaluation of Agreement in a Group of Experts via Distances between Intuitionistic Fuzzy Preferences" first international IEEE symposium intelligent systems, September 2002. 
[25]. E. Szmidt and J. Kacprzyk "Concept of Distances and Entropy for Intuitionistic Fuzzy Sets and their Applications in Group Decision Making" Sixth Int. Conf. on IFSs, Varna, NIFS 8 (2002), 3, 11-25.

[26]. D. Paternain, A. Jurio, E. Barrenechea, H. Bustince a, B. Bedregal, E. Szmidt "An alternative to fuzzy methods in decision-making problems", Expert Systems with Applications 39 (2012) 7729-7735.

[27]. E. Szmidt and J. Kacprzyk, "A Concept of Similarity for Intuitionistic Fuzzy Sets and its Use in Group Decision Making” IEEE International Conference 2004 Hungary vol.2 2004, 1129 - 1134.

[28]. Z. Xu, "Intuitionistic preference relations and their application in group decision making", Information Sciences: an International Journal archive, Volume 177 Issue 11, June, 2007 Pages 2363-2379.

[29]. Z. Xu, R. R. Yager, "Intuitionistic and interval-valued intuitionistic fuzzy preference relations and their measures of similarity for the evaluation of agreement within a group", Fuzzy Optimization and Decision Making Volume 8 Issue 2, June 2009123 - 139.

[30]. Z. Gong, "On the problem of group decision making based on intuitionistic fuzzy judgment matrices", Proceedings of the Life system modeling and simulation 2007, 315-325.

[31]. C. Wei and X. Tang, "An intuitionistic fuzzy group decision-making approach based on entropy and similarity measures", International Journal of Information Technology \& Decision Making (IJITDM), 2011, vol. 10, issue 06, pages 1111-1130.

[32]. Xu, Z and M. Xia, "Entropy/cross entropy-based group decision making under intuitionistic fuzzy environment”, Information Fusion, Volume 13 Issue 1, January, 2012, Page 31-47.

[33]. Zhi-xin Su, Guo-ping Xia, Ming-yuan Chen, Li Wang, "Induced generalized intuitionistic fuzzy OWA operator for multi-attribute group decision making”, Expert Systems with Applications Volume 39 Issue 2, 2012 Pages 1902-1910.

[34]. Y. Xu and H. Wang, "The induced generalized aggregation operators for intuitionistic fuzzy sets and their application in group decision making”, Applied Soft Computing March 2012, Volume 12 Issue 31168 1179.

[35]. C. Kahraman, "Fuzzy Multi-Criteria Decision Making: Theory and Applications with Recent Developments", Springer, Aug 19, 2008 - 591 pages.

[36]. H. Bustince, "Handling multicriteria fuzzy decision making problems based on intuitionistic fuzzy sets" Notes on IFS, Volume 1 (1995) Number 1, pages 42-47.

[37]. K. Atanassov, G. Pasi, and R. Yager "Intuitionistic Fuzzy Interpretations Of Multi-Person Multi-Criteria Decision Making" first international IEEE symposium "intelligent systems, September 2002.

[38]. G Pasi, R. Yager and K. Atanassov "Intuitionistic Fuzzy Graph Interpretations of Multi-Person MultiCriteria Decision Making: Generalized Net Approach", International IEEE Conference, Volume 2, June 2004, 434 - 439.

[39]. G Pasi, R. Yager and K. Atanassov "Intuitionistic fuzzy interpretation of multi-criteria multi-person and multi-measurement tool decision making", International Journal Of System Science vol 36. No 14, 15 November 2005, 859-868.

[40]. E.Szmidt and J. Kacprzyk, "An Application of Intuitionistic Fuzzy Set Similarity Measures to a Multicriteria Decision Making Problem", Lecture Notes in Computer Science, 2006, Volume 4029/2006, 314323.

[41]. S.M. Chen and J.M. Tan, "Handling multicriteria fuzzy decision-making problems based on vague set theory", Fuzzy Sets and Systems, 67(1994) 163-172.

[42]. L. Lin, XH. Yuan, ZQ. Xia, "Multicriteria fuzzy decision-making methods based on intuitionistic fuzzy sets", Journal of Computer and System Sciences 73 (2007) 84-88.

[43]. HW. Liu, G. Wang "Multi-criteria decision-making methods based on intuitionistic fuzzy sets", European Journal of Operational Research 179 (2007) 220-233.

[44]. C.Tan, Q. Zhang, Fuzzy Multiple Attribute Decision Making Based on Interval Valued Intuitionistic Fuzzy Sets, Systems, Man and Cybernetics, 2006. Volume 2, 2006, 1404- 1407.

[45]. Z. Xu, R. Yager, "Dynamic intuitionistic fuzzy multi-attribute decision making", International Journal of Approximate Reasoning 48 (2008) 246-262.

[46]. S. Y. Jie and W.F. Jie, "Dynamic multiattribute decision making based on the intuitionistic fuzzy priority rating model", ICMSE 2009- Management Science and Engineering, (2009) 239 - 245.

[47]. Jun Ye, "Application of correlation coefficient of interval-valued intuitionistic fuzzy sets to multicriteria fuzzy decision-making problems", Applied Mathematical Modeling (2008).

[48]. G. Wei and W. Yi, "Method for intuitionistic fuzzy multiple attribute decision making without weight information but with preference information on alternatives", Control and Decision Conference, (2008) $1970-1975$.

[49]. L. Dymova, I. Róg, P. Sevastjanov, "A New Method for Decision Making in the Intuitionistic Fuzzy Setting", Proceedings of the 9th international conference on Artificial Intelligence and Soft Computing $229-240$. 
[50]. L. Dymova, and P. Sevastjanov, "An interpretation of intuitionistic fuzzy sets in terms of evidence theory: Decision making aspect”, Knowledge-Based Systems, Volume 23 Issue 8 December, (2010) 772782.

[51]. Z. Wang; W. Wang; Lie, K.W., Multi-attribute decision making models and methods under intervalvalued intuitionistic fuzzy environment, Control and Decision Conference, (2008) 2420- 2425.

[52]. E. Szmidt, J. Kacprzyk "Ranking of Intuitionistic Fuzzy Alternatives in a Multi-criteria Decision Making Problem", 28th North American Fuzzy Information Processing Society Annual Conference (NAFIPS2009) 2009.

[53]. Z. Wang, J. Xu, W.Wang, "Intuitionistic fuzzy multiple attribute group decision making based on projection method", CCDC'09 Proceedings of the 21 st annual international conference on Chinese control and decision conference June 2009, 2967-2972.

[54]. Y. Luo, "Projection method for multiple attribute decision making with uncertain attribute weights under intuitionistic fuzzy environment", CCDC'09 Chinese control and decision conference (2009) 2993-2996.

[55]. Y. Luo, "Multiple attribute decision making with intuitionistic fuzzy information and uncertain attribute weights using minimization of regret", ICIEA 2009-4th IEEE Conference (2009)3720 - 3723.

[56]. J. Li and C. Zhang, "A New Solution of Intuitionistic Fuzzy Multiple Attribute Decision-Making Based on Attributes Preference", Fuzzy Systems and Knowledge Discovery, Volume 3(2009) 228 - 232.

[57]. H. Wang and G. Wei, "An Effective Supplier Selection Method with Intuitionistic Fuzzy Information" WiCOM '08-Wireless Communications, Networking and Mobile Computing,october 2008 pages $1-4$.

[58]. L. Wen and X. Zhang, "Intuitionistic Fuzzy Group Decision Making for Supply Chain Information Collaboration Partner Selection", ISME '10: Proceedings of the 2010 International Conference of Information Science and Management Engineering Volume 02 (2010) 43-46.

[59]. Y. Luo, Ye Jun, M. Xiaowen, 'Multicriteria fuzzy decision-making method based on weighted correlation coefficients under interval-valued intuitionistic fuzzy environment', Computer-Aided Industrial Design \& Conceptual Design, 2009. 2057- 2060.

[60]. L. webo, An enhanced multicriteria decision-making method of machine design schemes under intervalvalued intuitionistic fuzzy environment, Computer-Aided Industrial Design \& Conceptual Design, 2009, 721-725.

[61]. G. Wu and Wei, "GRA method for multiple attribute decision making with incomplete weight information in intuitionistic fuzzy", Knowledge-Based Systems, Volume 23 Issue 3, April, (2010) 243 247.

[62]. X. Feng and G. Qian, "The Method of Grey Related Analysis to Multiple Attribute Decision Making Problems with Intuitionistic Fuzzy Sets-Business and E-Government”, (ICEE), (2010), 1588 - 1591.

[63]. D.F. Li, "TOPSIS-Based Nonlinear-Programming Methodology for Multiattribute Decision Making With Interval-Valued Intuitionistic Fuzzy Sets' Fuzzy Systems, Volume:18, Issue: 2, 2010 ,299- 311.

[64]. Deng-Feng Li, Linear programming method for MADM with interval-valued intuitionistic fuzzy sets ,Expert Systems with Applications, Volume 37, Issue 8, August 2010, Pages 5939-5945

[65]. J.W. Wu and Q. Zhang, "Multicriteria decision making method based on intuitionistic fuzzy weighted entropy" Expert Systems with Applications Volume 38 Issue 1, January, 2011 Pages 916-922.

[66]. X. Yu, Z. Xu, Qi Chen, "A method based on preference degrees for handling hybrid multiple attribute decision making problems", Expert Systems with Applications, Volume 38 Issue 4, April, 2011, Pages 3147-3154.

[67]. Z. Zhitao; Z. Yingjun, "Multiple attribute decision making method in the frame of interval-valued intuitionistic fuzzy sets" Fuzzy Systems and Knowledge Discovery (FSKD)Volume:1 2011 ,192- 196

[68]. Z. Ying; Fan Ye; Jing Li; Zhen Jie Hong, Multiple attribute group decision making with incomplete information based on interval-valued intuitionistic fuzzy sets theory', Multimedia Technology (ICMT), 2011 , Page(s): 433- 436

[69]. Y. Ting Chen, H.P. Wang, Yen-Yu Lu, 'A multicriteria group decision-making approach based on interval-valued intuitionistic fuzzy sets', Expert Systems with Applications, Volume 38, Issue 6, June 2011, Pages 7647-7658.

[70]. G. Wei, X. Zhao, "Some induced correlated aggregating operators with intuitionistic fuzzy information and their application to multiple attribute group decision making", Expert Systems with Applications, Volume 39 Issue 2, February, (2012) 2026-2034.

[71]. Z. Xu and M. Xia, "Identifying and eliminating dominated alternatives in multi-attribute decision making with intuitionistic fuzzy information", Applied Soft Computing Volume 12 Issue 4, April, 2012 14511456.

[72]. Q. Jian Wang, K. Li, H. Zhang, 'Interval-valued intuitionistic fuzzy multi-criteria decision-making approach based on prospect score, Knowledge-Based Systems, Volume 27, March 2012, Pages 119-125. 